\title{
Blue-blocking intraocular implants should be used routinely during phacoemulsification surgery - Yes
}

Eye (2012) 26, 1397-1399; doi:10.1038/eye.2012.178; published online 7 September 2012

Cataract surgery removes a dysfunctional natural lens from the eye, replacing it with an artificial substitute. This substitute must mimic the role of a healthy lens in both focusing light onto the retina and protecting it from damage from electromagnetic radiation. The earliest intraocular lenses (IOLs) fulfilled only part of this requirement, being unable to block out harmful ultra-violet (UV) light. Contemporary clear IOLs do block UV light, but not short-wavelength visible light (blue light: 400-500 nm). ${ }^{1}$ In the phakic patient, the crystalline lens blocks out a proportion of blue light naturally with an increasing amount of blue light blocked with age. Replacing the crystalline lens with a UV-blocking IOL increases blue light transmission, and cataract surgeons are familiar with patients commenting that everything looks 'bluer' after surgery (cyanopsia). Blue-blocking IOLs more closely mimic the young crystalline lens, limiting that additional increase in blue-light transmission postoperatively.

In considering which IOL type to use, there are two important questions. Is additional retinal blue-light exposure harmful? Are there any advantages to allowing more blue light into a pseudophakic eye than would be possible even in a young, phakic eye?

That light exposure can cause retinal damage has been recognised for decades, with a number of different mechanisms identified. ${ }^{2,3}$ Both experimental and clinical evidence support that damage is caused to retinal and RPE cells by short, high-intensity light exposure, but the risk of long-term exposure to light of ambient levels remains under discussion. RPE cells in the ageing eye accumulate lipofuscin. This contains a fluorophore (A2E), which mediates RPE cell damage following short-wavelength light exposure in animal models and cell culture. ${ }^{4}$ This damage is reduced in the presence of a blue-light filter. ${ }^{1}$ What is less clear is how this theory translates into clinical practice.

Epidemiological studies have been inconsistent, with support for a role for light exposure in the development or progression of age-related macular degeneration (AMD) being seen in some but not all. ${ }^{1}$ A recent study linked light exposure to AMD development in a subgroup of patients with low macular-pigment levels, macular pigment having a putative protective role. ${ }^{5}$ This reflects the complex pathogenesis of AMD, with light exposure being just one of many possible contributing factors. There are significant logistical difficulties with such studies, which rely on a retrospective estimate of cumulative light exposure. AMD is a condition with multiple known and emerging risk factors, both genetic and environmental, and for any epidemiological study to be accurate, it must account for all. Because of the complexity of the condition, there is unlikely to be robust clinical or epidemiological evidence to support (or otherwise) the cell culture and animal model data soon; but in the meantime, the theoretical arguments for blue-light exposure causing retinal damage are strong. ${ }^{6}$

Use of a UV-blocking IOL increases blue-light transmission compared with both a young crystalline lens and a blue-blocking IOL. Is there any advantage to this? It has been argued that improved scotopic vision may be achieved in elderly patients. Rod function declines with age, ${ }^{7}$ and the rod sensitivity peak is approximately $500 \mathrm{~nm}$. Increasing the light 
input into these cells by removing a blue-light filter may improve vision. Although some theoretical studies have suggested improved scotopic sensitivity in eyes implanted with a clear, UV-blocking IOL compared with a blue-blocking IOL, ${ }^{8,9}$ two clinical studies failed to support this. ${ }^{10,11}$ In the industrialised world with its ambient lighting, true scotopic light levels are not often encountered, with street lighting and activities such as night driving falling into the low mesopic range. As such, even if there really was a small difference in performance under true scotopic conditions, it would be unlikely to cause significant difficulty to most people.

Blue light also has an important role in the circadian system, affecting the sleep-wake cycle, mood and cognition. ${ }^{12,13}$ This effect is mediated primarily by photoreceptive retinal ganglion cells (pRGCs) that contain the non-visual photopigment melanopsin and provide input to the hypothalamus. ${ }^{14}$ Melanopsin has a peak absorption wavelength of $480 \mathrm{~nm}$, at the blue end of the spectrum. The most important signal for circadian control is the changing quality of light at the extremes of the day, and in particular, exposure to morning daylight. Theoretical studies have suggested that a reduction in blue light transmission following implantation of a blue-blocking IOL could affect sleep. ${ }^{15}$ Clinical studies showed no difference between the two IOL types for sleep and quality of life, although numbers were small. ${ }^{16,17}$ To determine whether reduced blue transmission would affect sleep, it is important to consider absolute light levels and requirements. pRGCs act as integrators, meaning that duration of light exposure as well as intensity is important. A healthy, young adult requires $12 \mathrm{~h}$ of exposure to light of at least 200 lux (equivalent to average indoor lighting) to maintain circadian entrainment. ${ }^{18}$ Daylight is $2-3 \mathrm{log}$ units brighter than this, so even with a blue-blocking IOL, a short exposure to morning daylight should easily suffice for most individuals. Care should be taken, however, for those who do not have natural daylight exposure, such as those who are housebound or in nursing homes.

Blue-blocking IOLs aim to provide patients with a more physiological visual experience by blocking a proportion of blue light in the visible spectrum, equivalent to that which occurs naturally in a young, phakic adult. Although clinical evidence is still lacking, there is convincing theoretical and experimental evidence, suggesting that blue light may have the potential to damage the retina and possibly have a role in the pathogenesis of AMD. Suggested advantages of UV-blocking IOLs remain unproven. We believe that blue-blocking IOLs can be used safely in the majority of patients, and see no reason why they should not be used routinely. Every rule has an exception, however, and we would advocate caution in those with pre-existing sleep problems or who have poor daytime exposure to natural light.

\section{Conflict of interest}

RS declares no conflict of interest. FC has received funding to attend conferences from Allergan and Novartis.

\section{References}

1 Cuthbertson FM, Peirson SN, Wulff K, Foster RG, Downes SM. Blue light-filtering intraocular lenses: review of potential benefits and side effects. J Cataract Refract Surg 2009; 35: 1281-1297.

2 Youssef PN, Sheibani N, Albert DM. Retinal light toxicity. Eye 2011; 25: 1-14.

3 Van Norren D, Gorgels T. The action spectrum of photochemical damage to the retina: a review of monochromatic threshold data. Photochem Photobiol 2011; 87: 747-753.

4 Hunter JJ, Morgan JIW, Merigan WH, Sliney DH, Sparrow JR, Williams DR. The susceptibility of the retina to photochemical damage from visible light. Prog Retin Eye Res 2012; 31: 28-42.

5 Fletcher AE, Bentham GC, Agnew M, Young IS, Augood C, Chakravarthy U et al. Sunlight exposure, antioxidants, and age-related macular degeneration. Arch Ophthalmol 2008; 126: 1396-1403.

6 Jackson GRC, Owsley MGJ. Aging and dark adaptation. Vision Res 1999; 39: 3975-3982.

7 Margrain TH, Boulton M, Marshall J, Sliney DH. Do blue light filters confer protection against age-related macular degeneration? Prog Retin Eye Res 2004; 23: 523-531.

8 Pons A, Delgado D, Campos J. Determination of the action spectrum of the blue-light hazard for different intraocular lenses. J Opt Soc Am A Opt Image Sci Vis 2007; 24: 1545-1550.

9 Mainster MA, Sparrow JR. How much blue light should an IOL transmit? Br J Ophthalmol 2003; 87: 1523-1529.

10 Greenstein VC, Chiosi F, Baker P, Seiple W, Holopigian K, Braunstein RE et al. Scotopic sensitivity and color vision with a blue light-absorbing intraocular lens. J Cataract Refract Surg 2007; 33: 284-289.

11 Muftuoglu O, Karel F, Duman R. Effect of a yellow intraocular lens on scotopic vision, glare disability and blue color perception. J Cataract Refract Surg 2007; 33: 658-666.

12 Turner PL, Mainster MA. Circadian photoreception: ageing and the eye's important role in systemic health. $\mathrm{Br} \mathrm{J}$ Ophthalmol 2008; 92: 1439-1444.

13 Mainster MA, Turner PL. Blue-blocking IOLs decrease photoreception without providing significant photoprotection. Surv Ophthalmol 2010; 55: 272-283.

14 Hankins MW, Peirson SN, Foster RG. Melanopsin: an exciting new photopigment. Trends Neurosci 2008; 31: 27-36. 
15 Mainster MA. Violet and blue light blocking intraocular lenses: Photoprotection versus photoreception. $\mathrm{Br} \mathrm{J}$ Ophthamol 2006; 90: 784-792.

16 Landers JA, Tamblyn D, Perriam D. Effect of a blue-lightblocking intraocular lens on the quality of sleep. J Cataract Refract Surg 2009; 35: 83-88.

17 Espindle D, Crawford B, Maxwell A, Rajagopalan K, Barnes R, Harris B et al. Quality-of-life improvements in cataract patients with bilateral blue light-filtering intraocular lenses: clinical trial. J Cataract Refract Surg 2005; 31: 1952-1959.

18 Middleton B, Stone BM, Arendt J. Human circadian phase in 12:12 h, 200: $<8$ lux and 1000: $<8$ lux light-dark cycles, without scheduled sleep or activity. Neurosci Lett 2002; 329: 41-44.
RJ Symes ${ }^{1}$ and FM Cuthbertson ${ }^{2}$

${ }^{1}$ Department of Ophthalmology, Sussex Eye Hospital, Brighton, UK and ${ }^{2}$ Royal United Hospital Bath NHS Trust, Bath, UK

Correspondence: RJ Symes, Department of Ophthalmology, Sussex Eye Hospital, Eastern Road, Brighton BN2 5BF, UK Tel: + 44 (0) 1273 606126; Fax: + 44 (0) 1273553038. E-mail: richsymes@hotmail.com 